\title{
Cognitive Electrophysiology Assessment
}

National Cancer Institute

\section{Source}

National Cancer Institute. Cognitive Electrophysiology Assessment. NCI Thesaurus. Code C156776.

Assessments of cognition that focus on intracranial recordings of brain activity. 